\title{
The Role of Sputtering Current on the Optical and Electrical Properties of Si-C Junction
}

\author{
Mohammad M. Uonis, Bassam M. Mustafa, Anwar M. Ezzat* \\ Department of Physics, College of Science, Mosul University, Mosul, Iraq \\ Email: ${ }^{*}$ prof.dr.anwar@uomosul.edu.iq
}

Received 21 April 2014; revised 19 May 2014; accepted 19 June 2014

Copyright $@ 2014$ by authors and Scientific Research Publishing Inc.

This work is licensed under the Creative Commons Attribution International License (CC BY).

http://creativecommons.org/licenses/by/4.0/

(c) (i) Open Access

\begin{abstract}
The effect of sputtering current that flow in a carbon rod on the structural and transport properties of Si-C junction is studied. Si-C junction is fabricated by plasma sputtering in Argon gas atmosphere without catalysts with thickness of 20, 40 and $60 \mathrm{~nm}$. Images of the specimen by scanning electron microscope (SEM) and atomic force microscope (AFM) show that the carbon layer is as carbon nanotubes with diameters about $20-30 \mathrm{~nm}$. X-ray and Raman spectrums show peak characteristics of the carbon nanotubes, the $G$ and $D$ bands appear for all thicknesses indicating free of defect carbon nanotubes. Two parameters about the thickness of the carbon layer and the sputtering current for different thicknesses and currents were studied. Nanotubes evidence was clear. We noticed that the sputtering current and thickness of layers affect the structure of CNT layer leading to the formation of grains. Increasing plasma current led to decrease grain formation however increasing thickness ends to increase grain size; moreover it led to amorphous structure formation and this was proved through X-ray, Raman spectra and AFM images.
\end{abstract}

\section{Keywords}

Carbon Nanotubes, SEM, AFM and X-Ray Multi Wall Images, Plasma Sputtering and Si-C Junction

\section{Introduction}

Carbon Nanotubes (CNT's) are cylindrical structures of nanoscale diameter; it composed of carbon atoms in a hexagonal arrangement. They were discovered in 1991 by Japanese scientist Sumio Iijima [2]. The unique properties of CNT's, such as tremendous strength and excellent thermal and electrical conductivity, have caused this

*Corresponding author.

How to cite this paper: Uonis, M.M., Mustafa, B.M. and Ezzat, A.M. (2014) The Role of Sputtering Current on the Optical and Electrical Properties of Si-C Junction. World Journal of Nano Science and Engineering, 4, 90-96.

http://dx.doi.org/10.4236/wjnse.2014.42012 
material to become the focus of intense research by many groups [1]-[3].

There are three commonly used methods of CNT synthesis. Arc-discharge method employs evaporation of graphite electrodes in electric arcs that involve very high temperatures $\left(\sim 4000^{\circ} \mathrm{C}\right)$. Laser-vaporization technique employs evaporation of high-purity graphite target by high-power lasers in conjunction with high-temperature furnaces. Chemical vapor deposition (CVD) required incorporating catalyst-assisted by thermal decomposition of hydrocarbons [1]-[5].

All the above methods need catalysts such as $\mathrm{Ni}, \mathrm{Fe}$...etc. In our research, we use plasma scattering of carbon rod to prepare Si-C junction, which was studied earlier thoroughly in our published paper [6].

In this paper, we want to find the effect of changing the sputtering current and the thickness effect on the structural and electrical properties of the CNT layer in the Si-C junction.

\section{Experimental Method}

We report the synthesis of carbon nanotubes on silicon wafers without catalyst of large aspect ratios by plasma sputtering method as shown in Figure 1. The carbon-containing and reacting gases introduced into the chamber through a network with mass flow controllers allowing to regulate the flow rate and gas composition of the mixture. A high voltage applied to the electrode above the sample causes an ionization of the gases, resulting in plasma formation. This method allows the control of growth parameters to influence growth rateand diameter.

The two most important features of this technique are carbon layer thickness and current flow through the carbon rod, which studied using scanning electron microscopy (SEM), Atomic force microscope (AFM), X-ray diffraction (XRD) and Raman spectroscopy.

The surface microstructure obtained by a cold field scanning electron microscope (SEM) (JEUM-JSM-6756 F) operating at a voltage of $10 \mathrm{keV}$. Scanning electron microscope (Jeol; JDS-7391LV) was used to record Energy dispersive scattering (EDS).

Raman spectra measurements (GM SER No 87,120) in (Germany made). The current voltage characteristics obtained using A WXS140s Wacom sun simulator.

Micro-Raman spectroscopy consists of a laser excitation beam that focused through a microscope on the sample surface. The back scattered light intensity is measured as a function of its frequency shift. These shifts induced by the inelastic energy exchange between photons and vibration modes. The spectra obtained give information on the bonding environment in the sample. X-ray diffraction (XRD, Bruker/D8-advance with $\mathrm{Cu} \mathrm{K} \alpha$ radiation $(\lambda=1.54178 \AA)$, the diffraction angle was scanned from $20 \pm$ to $100 \pm$ at the scanning speed of 0:02 \pm per second.

\section{The Results}

Many measurements were done include Scanning electron microscopy (SEM), Atomic force microscope (AFM), X-ray diffraction (XRD) and Raman spectra are taken.

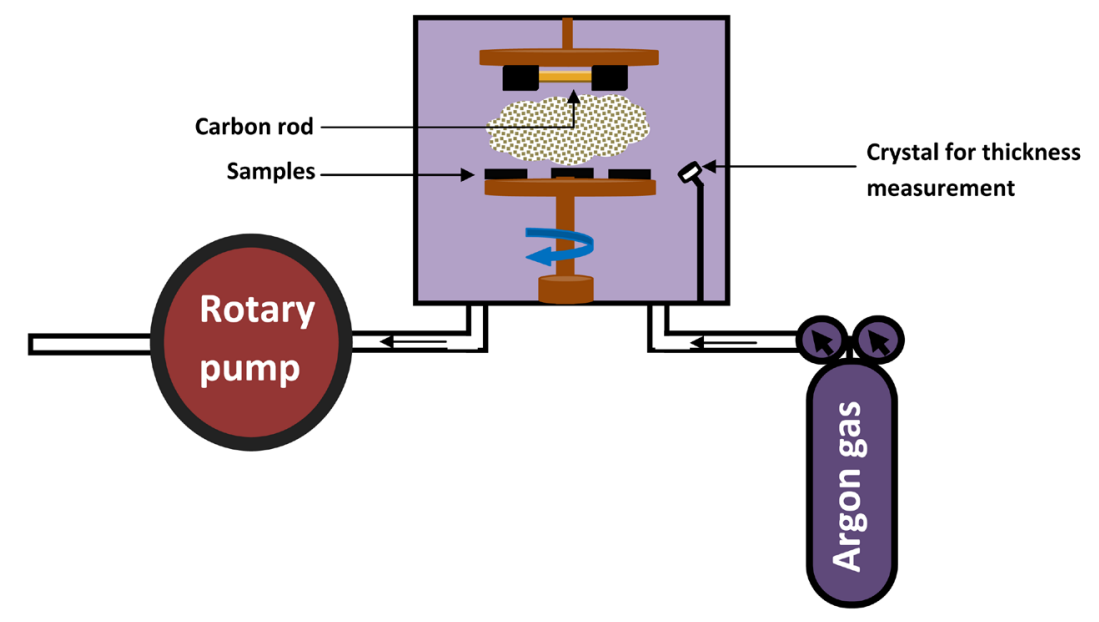

Figure 1. Schematic of plasma sputtering system. 
Two parameters were studied, the thickness of the carbon layer and the sputtering current. For all thicknesses and all currents included in the study, the effect of plasma sputtering current is opposite to increasing of layers thickness. Increasing plasma current will decrease grain formation and increasing thickness will increases grain size and moreover it lead to amorphous structure formation, this were proved by X-ray, Raman spectra and AFM images.

Carbon layers deposited using 70 A current in the carbon rod are shown in Figures 2(a)-(c). From Figure 2(a), it is noticed that the sputtering process produces small grains and these grains grows with thickness increase as shown in Figure 2(b) and Figure 2(c) for 40 and $60 \mathrm{~nm}$ respectively.

In Figure 2(c), clearly shown bigger grains due to cohesion between the small grains as a result of the continued growth in their dimensions with increasing the thickness of the carbon layer.

In Figure 2(a), Figure 2(d) and Figure 2(g), represents SEM images due to sputtering currents in the carbon layer with values 70, 80 and 90 A with constant thickness $(20 \mathrm{~nm})$. The increase in current flow in the carbon rod produces further excitation(increase in the energy of plasma) in plasma which tries to obstruct the formation of grains and this will lead to produce more amorphous phase at the expense of carbon nanotubes formation (ordered phase) in the deposited carbon layer.

As a conclusion, the increase of thickness will produce grains that grown slowly with high currents. This agree with the results of $\mathrm{X}$-ray and Raman spectra, which will be discussed later.

Raman spectra revealed the G and D bands clearly for all thicknesses and just for $70 \mathrm{~A}$ and 80 A. However, for high current $90 \mathrm{~A}$, the $\mathrm{G}$ and $\mathrm{D}$ bands distorted as shown in Figure 3.

From Figure 3(a) and Figure 3(b), the most important features seen are the disorder induced D band at 1340 $-1350 \mathrm{~cm}^{-1}$ and the tangential G band at $1550-1580 \mathrm{~cm}^{-1}$, which is related to the graphite tangential Raman active mode where the two atoms in graphene unit cell are vibrating tangentially one against the other. A presence in the Raman spectra of D bands with the frequency $1350 \mathrm{~cm}^{-1}$ is ascribed to a presence of amorphous Carbon in the sample, while the presence of $\mathrm{G}$ bands with a frequency $1580 \mathrm{~cm}^{-1}$ give us information about the existence of ordered Carbon structure [7]-[12].

We note that the specific peaks shown for $70 \mathrm{~A}$ and $80 \mathrm{~A}$ are clear but they decrease when increasing current to $90 \mathrm{~A}$. The Raman spectrum distorted due to the increase in plasma energy by increasing current.

The ratio of $\mathrm{I}_{\mathrm{D}} / \mathrm{I}_{\mathrm{G}}$ bands (about $74 \%$ for $70 \mathrm{~A}$ and $77 \%$ for $80 \mathrm{~A}$ ) give indication that the more probable phase is the ordered mode, but for 90 A power, the amorphous mode increase as a result of high sputtering power, so that we conclude that the amorphous mode will increase with increasing of flow current, this conclusion matches with conclusion that drown from the SEM images [10].
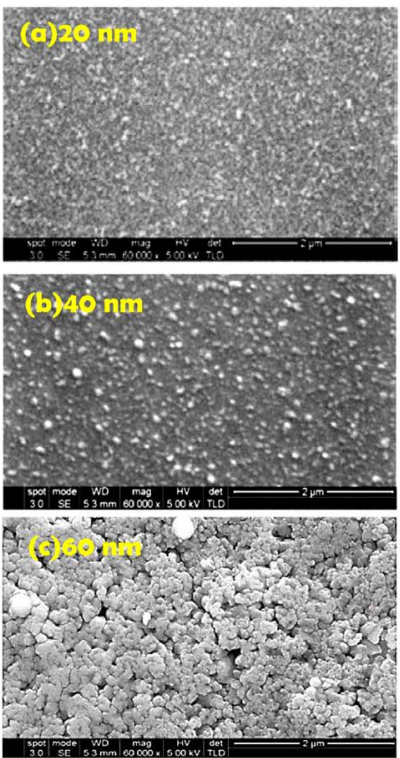

Current 70 A
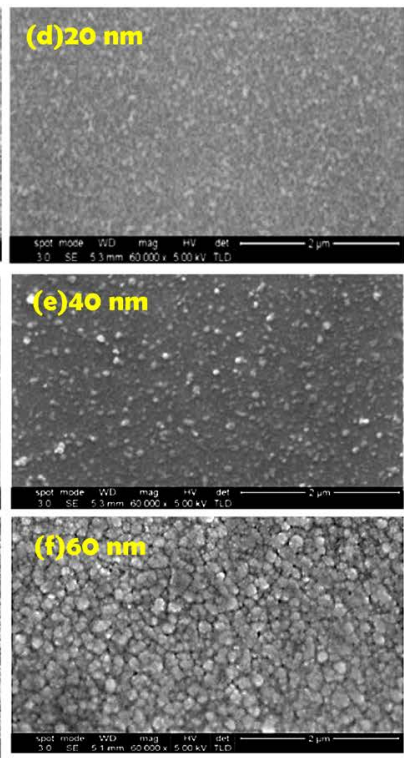

Current 80 A
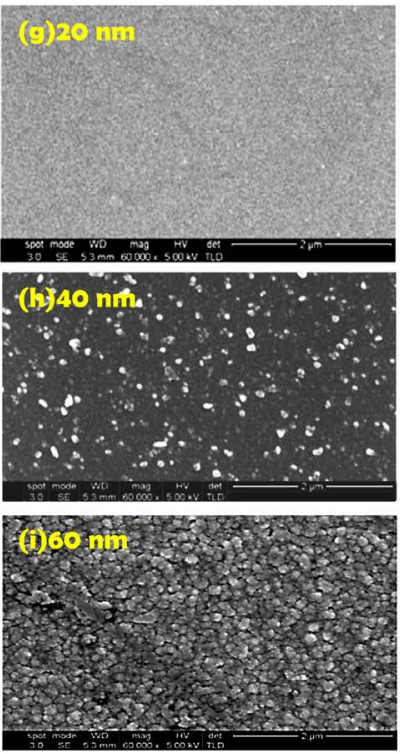

Current 90 A

Figure 2. SEM images of Carbon layers for a range of currents $70 \mathrm{~A}, 80 \mathrm{~A}$ and $90 \mathrm{~A}$. 


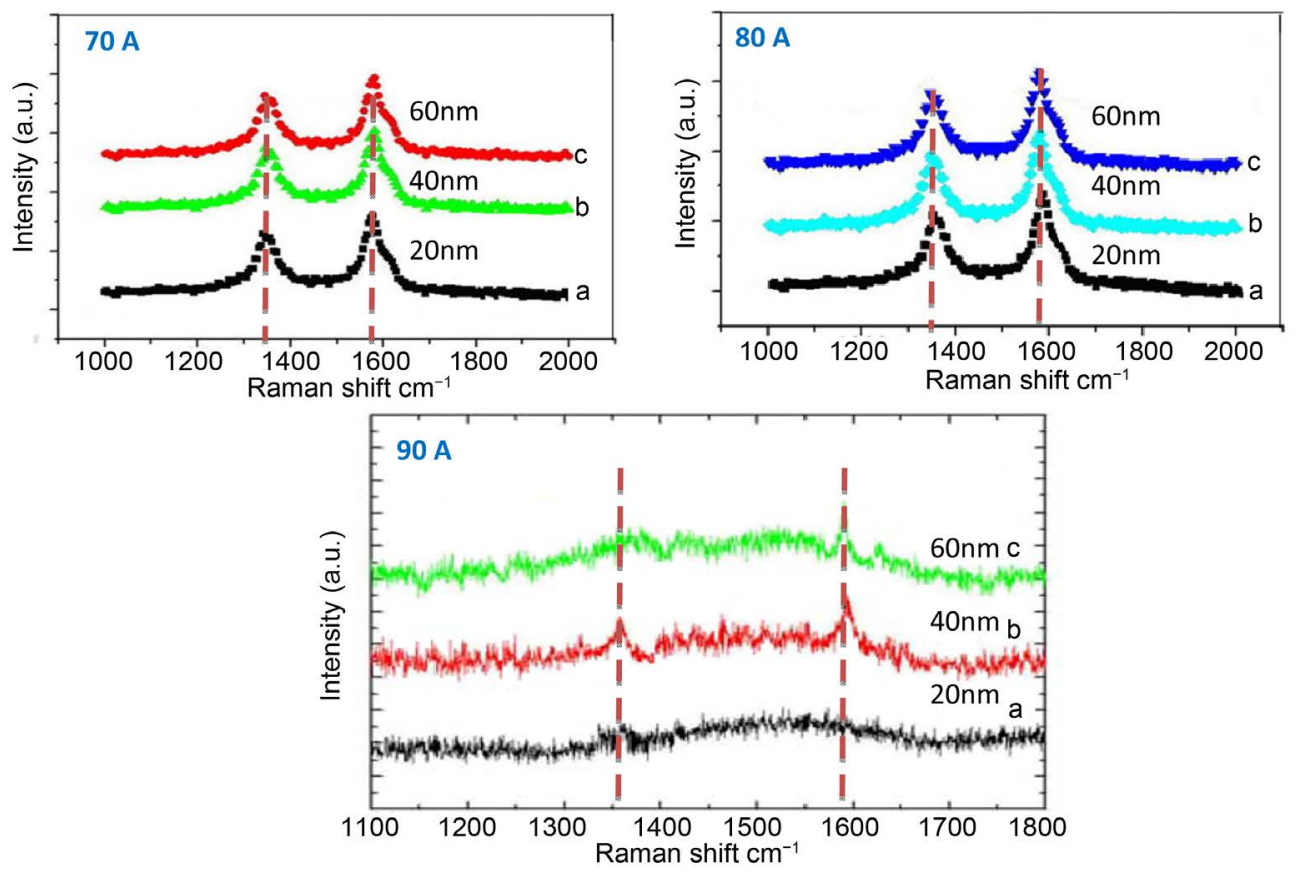

Figure 3. The Raman spectra of Carbon layers for a range of plasma currents $70 \mathrm{~A}, 80 \mathrm{~A}$ and $90 \mathrm{~A}$.

The Raman features associated with the radial breathing mode (RBM) from the large diameter tubes is usually too poor to be observable. The D band activated in the first order scattering process of sp2 Carbons by the presence of in plane substitution heteroatom vacancies, grain boundaries, or other defects and by finite size effects, all of which lower the crystalline symmetry of the quasi-infinite lattice. Therefore, the D mode could be used as a diagnostic of disruptions in the hexagonal framework of MWNTs and is induced by double resonance process. This result also observed elsewhere [10].

The shape of the G Raman peak gives possibility to distinguish between semiconducting or metallic nanotubes. Here, we think, that the nanotubes are associated with semiconducting type of conductivity as mentioned in ref. [12].

In purified sample $\mathrm{G}$ band is narrower than in non-purified, so the spectra shows that our samples were well purified. At low thickness the intensity is somewhat lower than that of high thickness, this indicate that the diameter of the nanotube is increasing, this is clear through comparing (a) with (b) and (c) spectra in Figure 2.

$\mathrm{X}$-ray measurements for Carbon nanotubes is somewhat different from that of crystalline or polycrystalline samples. X-ray diffraction of CNT gives indication of the presence of graphite layers. X-ray diffraction pattern shows clearly the characteristic peak (002) of the graphite layers which indicate surly the existence of multiwall Carbon nanotube. From Figure 4, it can be observed that the peaks for power 70 A Can be specified clearly for all thicknesses and they are distorted with increasing sputtering current due to increase in amorphous phase, In addition, the increase in thickness is inversely proportional to spectrum distortion for $80 \mathrm{~A}$ and $90 \mathrm{~A}$ currents, also it is noted that some peaks which belong to Si-C are disappeared in high current spectrums. XRD spectrum also shows characteristic peaks of $\mathrm{Si}$, which belong to the substrate, and $\mathrm{Si}-\mathrm{C}$, which is normally formed at the junction but the intensity, is low indicating small quantity of Si-C.

Figure 5 shows the atomic force microscope AFM images of the carbon layer on silicon wafer for different thicknesses and powers. It can be observed that the surface roughness of the carbon layers increases with thickness for all currents, this roughness is due to the formation of grains and their growth, so that the amorphous mode (grains) and short CNTS were clearly shown in this figure which increases with thickness.

In Figure 6, the three dimensional images of the samples for 90 A current are shown. It is clear that there is an enlargement in the grain size with increasing thickness.

Figures 7(a)-(c) show the I-V characteristics for the Si-CNT junction for all currents and all thicknesses under study, it is noticed that the current decreases with sample thickness for the same current in the carbon rod. Moreover for the same thickness, the current in I-V characteristics increases with the current flows in the carbon 

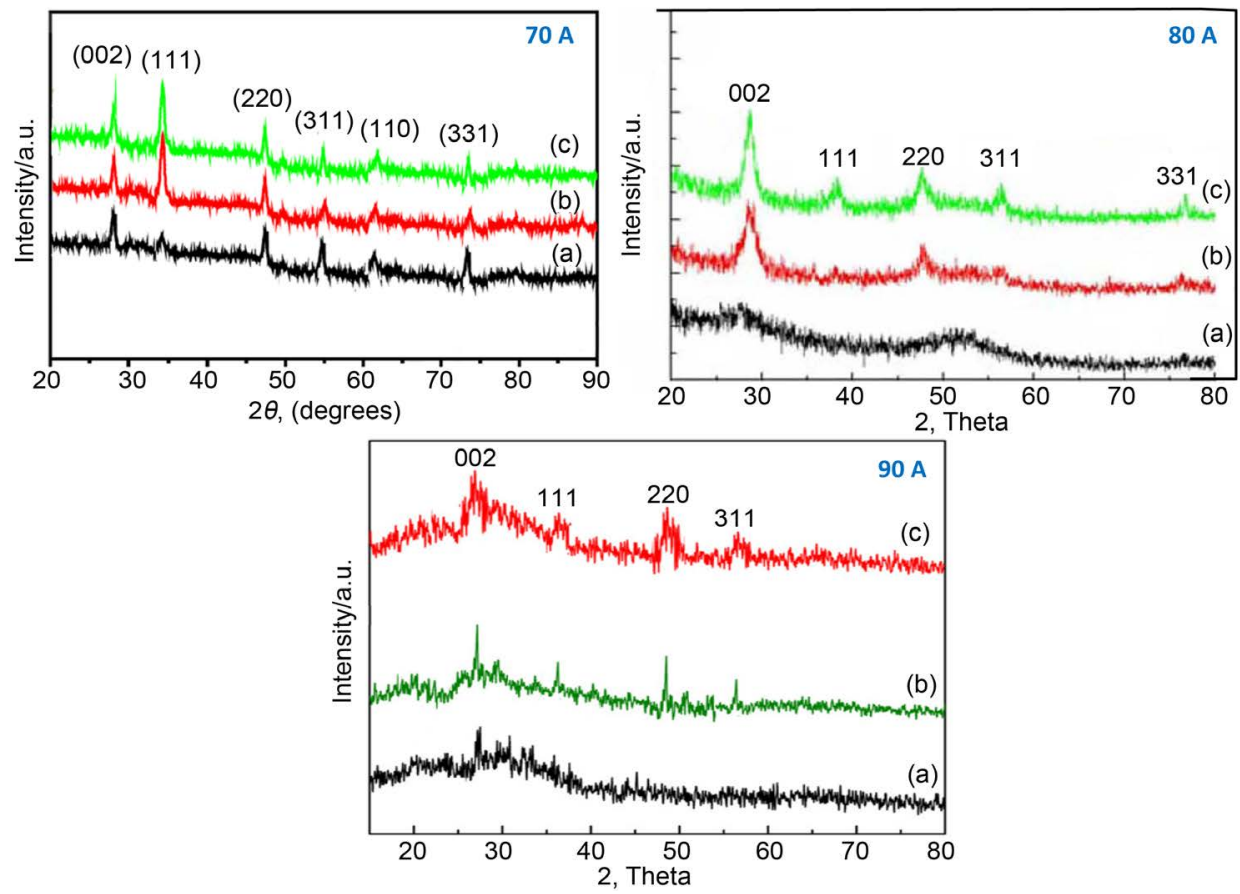

Figure 4. X-ray spectrum for Carbon layers (a) $20 \mathrm{~nm}$ thickness, (b) $40 \mathrm{~nm}$ thickness and (c) 60 nm thickness.
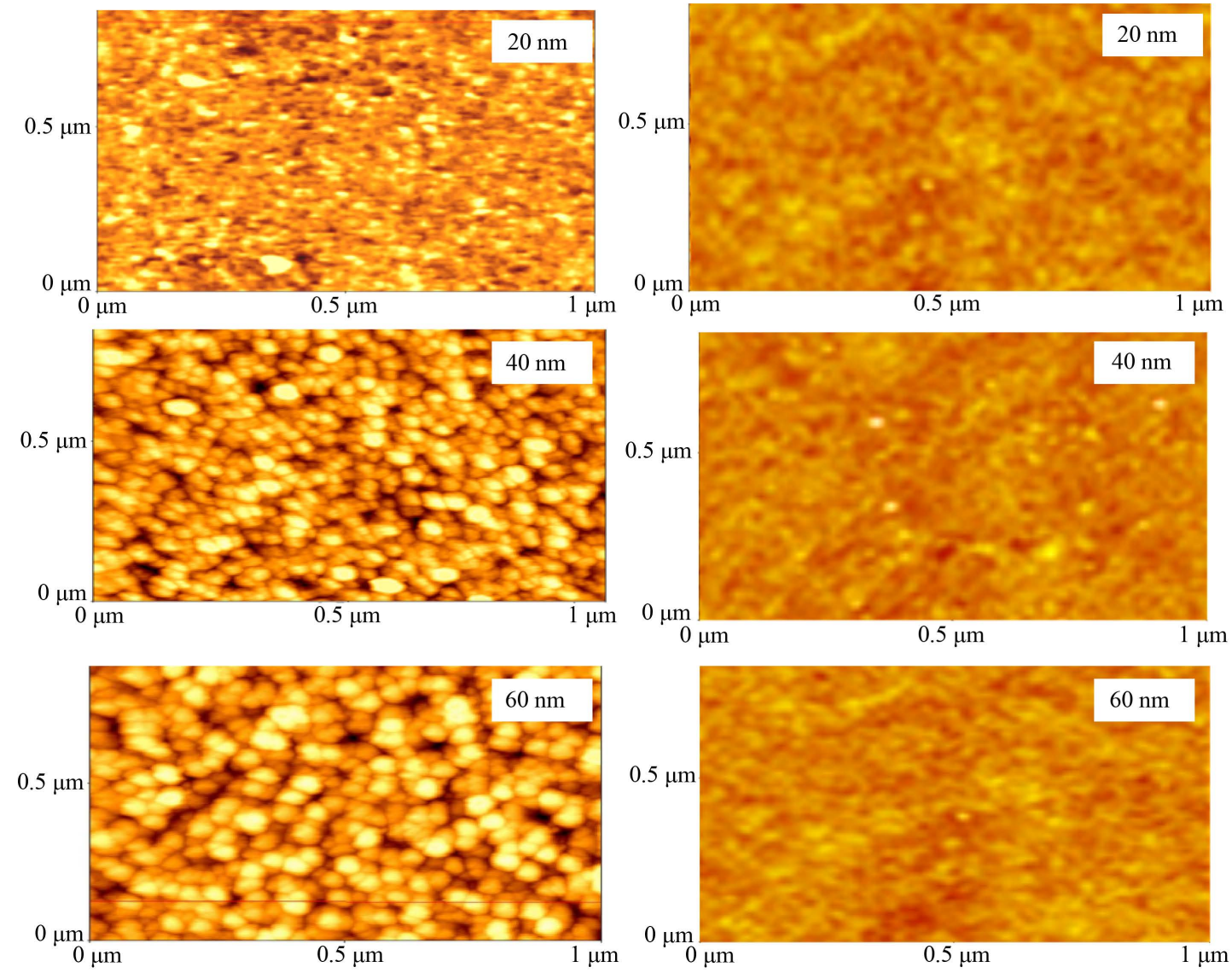

Figure 5. AFM images of Carbon layers for a range of currents $70 \mathrm{~A}, 80 \mathrm{~A}$ and $90 \mathrm{~A}$. 

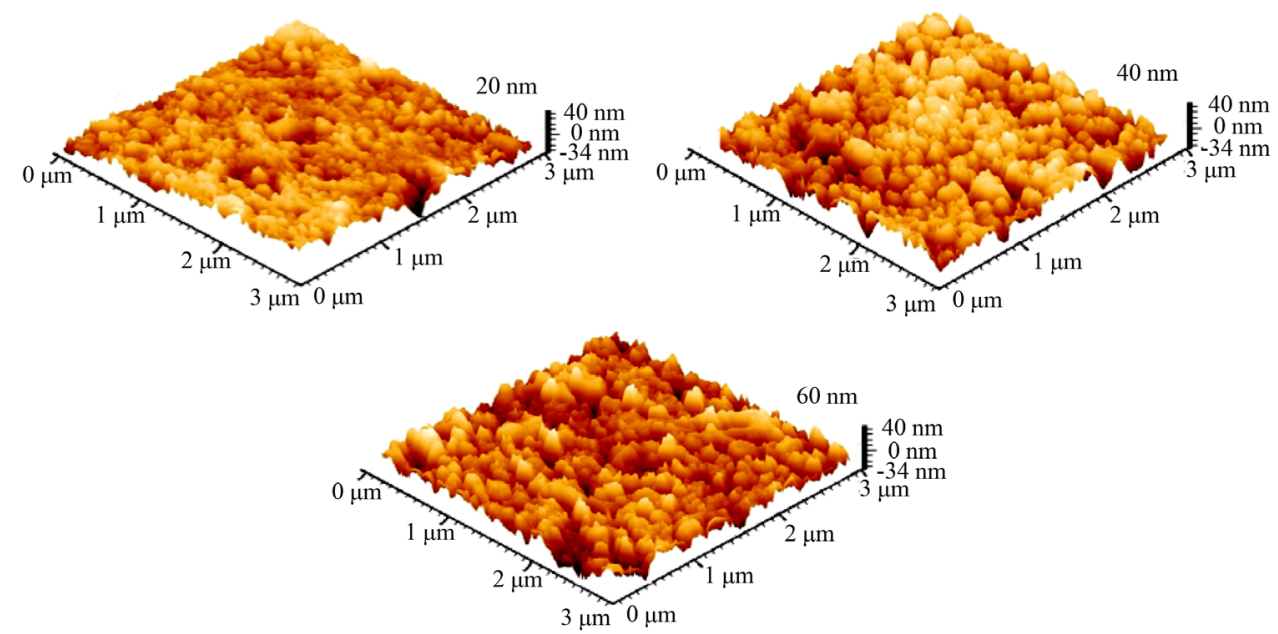

Figure 6. AFM images of Carbon layers for plasma current $90 \mathrm{~A}$.

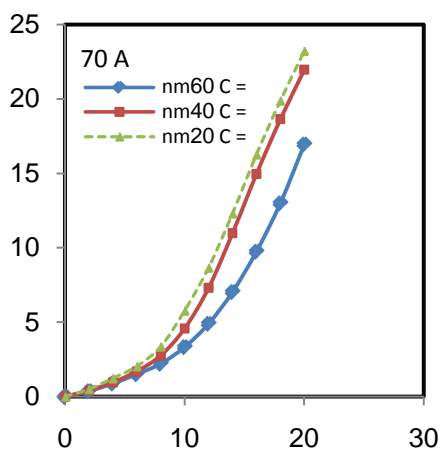

(a)

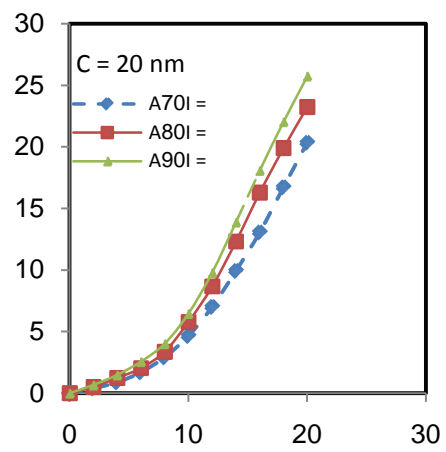

(d)

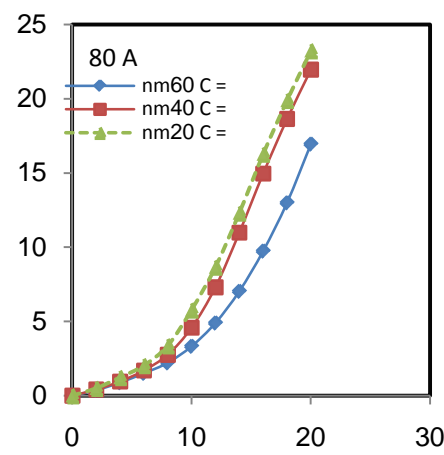

(b)

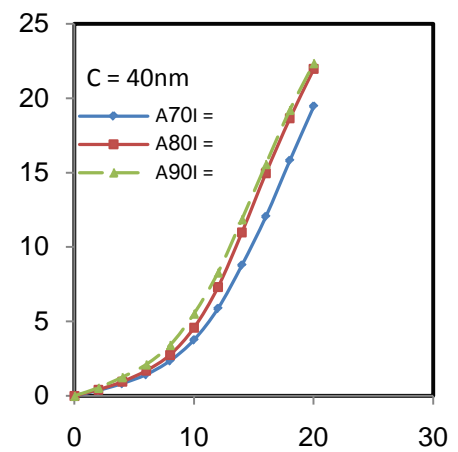

(e)

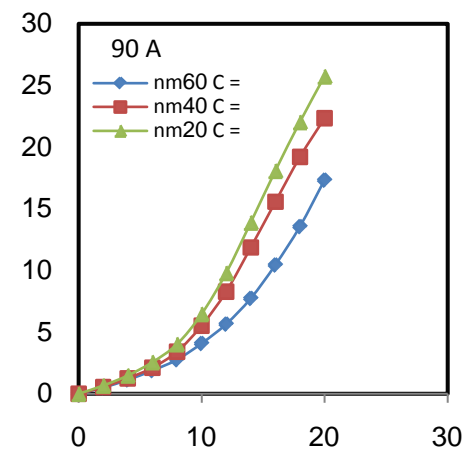

(c)

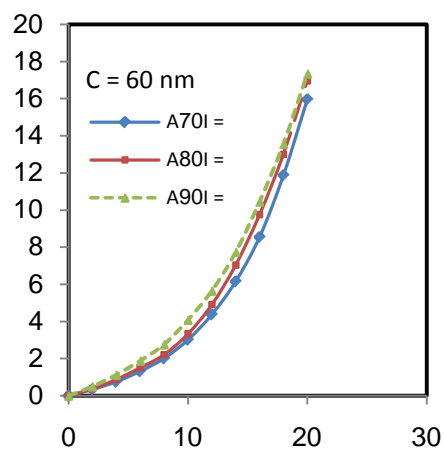

(f)

Figure 7. The I-V characteristics of Si-CNT junction for different plasma current and thicknesses.

rod as in Figures 7(a)-(c). As a general behavior of the junction, I-V characteristics show semiconducting behavior.

For constant thickness, the increase in the plasma current flowing in the carbon rod will increase the energy of the plasma, which pervert the formation of the grains in the deposited carbon layer, reduces the amorphous phase, and thus reduces distortions occurring in this layer, which in turn is reflected in the increase in I-V characteristics current.

\section{Conclusions}

1) The joined effect of increasing thickness of the CNT layer in Si-CNT junction and the sputtering current in 
the carbon rod led to the formation of grains.

2) At certain sputtering current increasing thickness of the layer increases the grain size.

3) Increasing sputtering current that flows in the carbon rod led to increase the plasma energy, which lead to increase percentage of amorphous phase in the CNT layer.

4) I-V characteristic shows semiconducting behavior and the current decreases with increasing thickness for the same sputtering current in the carbon rod, whereas the current increases with increasing sputtering current in the carbon rod.

\section{Acknowledgements}

The Authors would like to thank the College of Science, administration at Mosul University for supporting this work. Also thanks to Abid Al Karem M Muhammed (Sumysyate University/Department of Nano/Ukraine) for his great help in samples measurements.

\section{References}

[1] Valentini, L., Armentano, I., Kenny, J.M., Lozzi, L. and Santucci, S. (2003) Pulsed Plasma-Induced Alignment of Carbon Nanotubes. Materials Letters, 57, 3699-3704. http://dx.doi.org/10.1016/S0167-577X(03)00166-6

[2] Lehman, J.H., Terrones, M., Mansfield, E., Hurst, K.E. and Meunier, V. (2011) Evaluating the Characteristics of Multiwall Carbon Nanotubes. Carbon, 49, 2581-2602. http://dx.doi.org/10.1016/j.carbon.2011.03.028

[3] Hofmann, S., Kleinsorge, B., Ducati, C., Ferrari, A.C. and Robertson, J. (2004) Low-Temperature Plasma Enhanced Chemical Vapor Deposition of Carbon Nanotubes. Diamond and Related Materials, 13, 1171-1176. http://dx.doi.org/10.1016/j.diamond.2003.11.046

[4] Choi, Y.C., Bae, D.J., Lee, Y.H. and Lee, B.S. (2000) Growth of Carbon Nanotubes by Microwave Plasma-Enhanced Chemical Vapor Deposition at Low Temperature. Journal of Vacuum Science \& Technology A, 18, 1864-1868.

[5] Muratore, C., Reed, A.N., Bultman, J.E., Ganguli, S., Cola, B.A. and Voevodin, A.A. (2013) Nanoparticle Decoration of Carbon Nanotubes by Sputtering. Carbon, 57, 274-281. http://dx.doi.org/10.1016/j.carbon.2013.01.074

[6] Ezzat, A.M., Mustafa, B.M. and Uonis, M.M. (2014) Fabrication of Si-CNT Junction by Plasma Sputtering of Graphite Rods on Silicon Wafers. International Journal of Advanced Research, 2, 108-113.

[7] Costa, S., Borowiak-Palen, E., Kruszyńska, M., Bachmatiuk, A. and Kaleńczuk, R.J. (2008) Characterization of Carbon Nanotubes by Raman Spectroscopy. Materials Science-Poland, 26, 433-441.

[8] Bokova, S.N., Obraztsova, E.D., Grebenyukov, V.V., Elumeeva, K.V., Ishchenko, A.V. and Kuznetsov, V.L. (2010) Raman Diagnostics of Multi-Wall Carbon Nanotubes with a Small Wall Number. Physica Status Solidi (B), 247, 2827-2830. http://dx.doi.org/10.1002/pssb.201000237

[9] Jorio, A., Pimenta, M.A., Souza Filho, A.G., Saito, R., Dresselhaus, G. and Dresselhaus, M.S. (2003) Characterizing Carbon Nanotube Samples with Resonance Raman Scattering. New Journal of Physics, 5, 139.1-139.17.

[10] Murphy, H., Papakonstantinou, P. and Okpalugo, T.I.T (2006) Raman Study of Multiwalled Carbon Nanotubes Functionalized with Oxygen Groups. Journal of Vacuum Science \& Technology B, 24, 715.

[11] Zdrojek, M., Gebicki, W., Jastrzebski, C., Melin, T. and Huczko, A. (2004) Studies of Multiwall Carbon Nanotubes Using Raman Spectroscopy and Atomic Force Microscopy. Solide State Phenomena, 99, 265.

[12] Bellucci, S., Gaggiotti, G., Marchetti, M., Micciulla, F., Mucciato, R. and Regi, M. (2007) Atomic Force Microscopy Characterization of Carbon Nanotubes. Journal of Physics: Conference Series, 61, 99-104. 
Scientific Research Publishing (SCIRP) is one of the largest Open Access journal publishers. It is currently publishing more than 200 open access, online, peer-reviewed journals covering a wide range of academic disciplines. SCIRP serves the worldwide academic communities and contributes to the progress and application of science with its publication.

Other selected journals from SCIRP are listed as below. Submit your manuscript to us via either submit@scirp.org or Online Submission Portal.
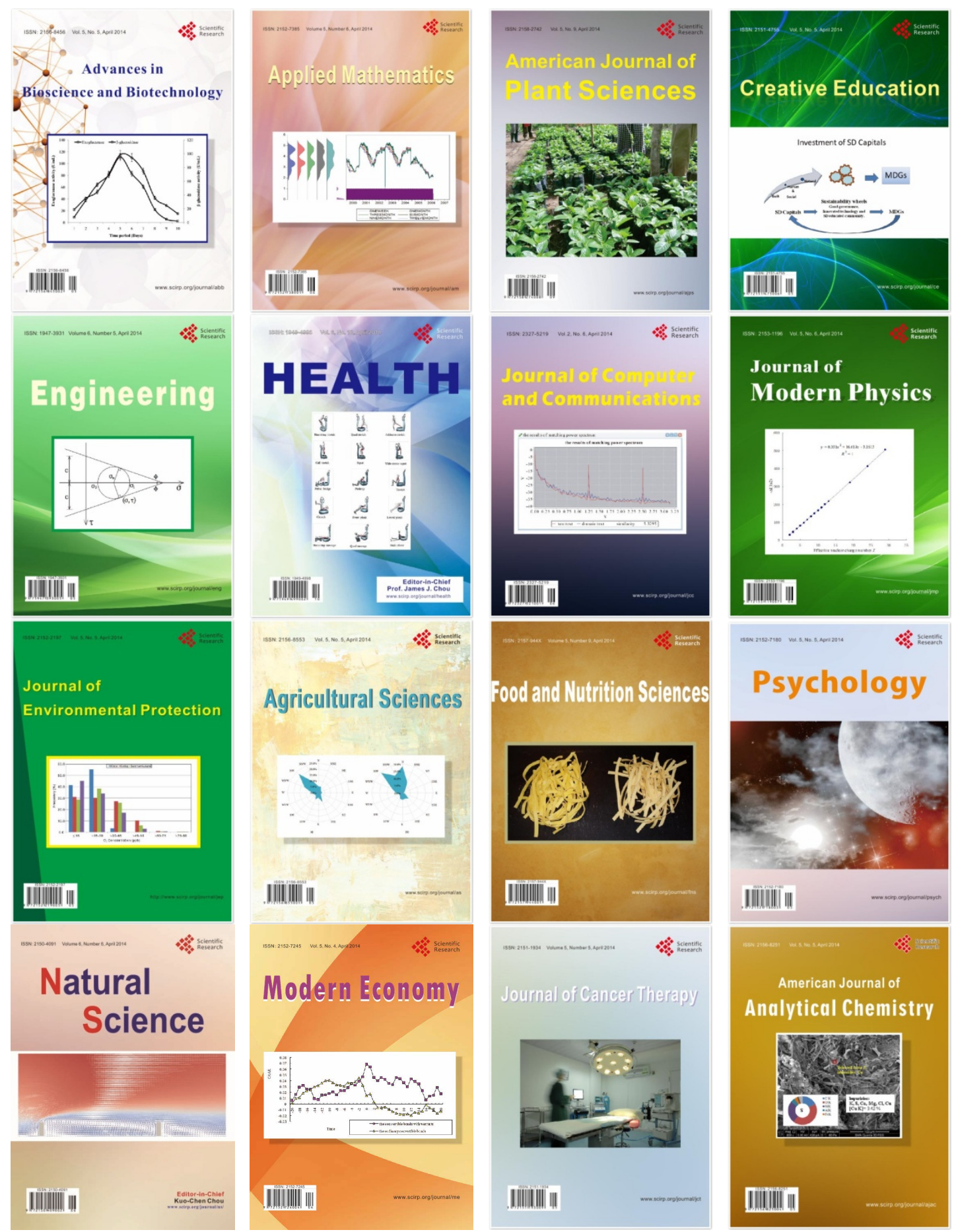